Modern Physics Letters A

(C) World Scientific Publishing Company

\title{
NERNST HEAT THEOREM FOR THE CASIMIR-POLDER INTERACTION BETWEEN A MAGNETIZABLE ATOM AND FERROMAGNETIC DIELECTRIC PLATE
}

\author{
C. C. KORIKOV ${ }^{1}$ and V. M. MOSTEPANENKO ${ }^{1,2,3}$ \\ ${ }^{1}$ Institute of Physics, Nanotechnology and Telecommunications, Peter the Great \\ Saint Petersburg Polytechnic University, Saint Petersburg, 195251, Russia \\ ${ }^{2}$ Central Astronomical Observatory at Pulkovo of the Russian Academy of Sciences, \\ Saint Petersburg, 196140, Russia \\ ${ }^{3}$ Kazan Federal University, Kazan, 420008, Russia \\ constantine.korikov@gmail.com,vmostepa@gmail.com
}

Received 12 July 2019

Revised 8 August 2019

\begin{abstract}
We find the low-temperature behavior of the Casimir-Polder free energy for a polarizable and magnetizable atom interacting with a plate made of ferromagnetic dielectric material. It is shown that the corresponding Casimir-Polder entropy goes to zero with vanishing temperature, i.e., the Nernst heat theorem is satisfied, if the dc conductivity of the plate material is disregarded in calculations. If the dc conductivity is taken into account, the Nernst theorem is violated. These results are discussed in light of recent experiments.
\end{abstract}

Keywords: Casimir-Polder free energy; Nernst heat theorem; ferromagnetic dielectrics.

PACS Nos.: 12.20.Ds, 34.35.+a, 65.40.gd

\section{Introduction}

The Nernst heat theorem has long been used as a test for different theoretical approaches to the Casimir force. Thus, in Ref. 1 the behavior of the Casimir free energy and entropy at low temperature was found in the configuration of two parallel ideal metal plates. It was proven that at zero temperature the Casimir entropy is equal to zero in accordance with the Nernst theorem. At a later time, it was shown that for two metallic plates with perfect crystal lattice the Casimir entropy satisfies the Nernst theorem if the low-frequency dielectric response of metal is described by the lossless plasma model and violates this theorem if the lossy Drude model is used.$^{2 \sqrt{5}}$ The same is true for a metallic sphere and a plate ${ }^{6}$ and for two plates made of ferromagnetic metal. ${ }^{7}$ For an atom interacting with metallic plate the Nernst theorem is followed regardless of the used model of dielectric response of metal if this atom does not possess magnetic properties. For a magnetizable atom, however, the Casimir-Polder entropy satisfies and violates the Nernst theorem when the plasma and Drude models are used, respectively, like for two metallic plates ${ }^{8}$ 
For two dielectric plates described with omitted conductivity at a constant current (dc conductivity), the Casimir entropy satisfies the Nernst theorem and violates it otherwise. ${ }^{911}$ The same holds for two plates made of ferromagnetic dielectric ${ }^{12}$ and for the Casimir-Polder entropy of a polarizable atom interacting with nonmagnetic dielectric plate ${ }^{13}$ Thus, for a dielectric plate, thermodynamic properties depend on the used model of dielectric response even if an atom is not magnetizable.

In this paper, we find an analytic expression for the Casimir-Polder free energy at low temperature in more general case of polarizable and magnetizable atom interacting with a plate made of ferromagnetic dielectric. We show that the respective Casimir-Polder entropy satisfies the Nernst theorem if the dc conductivity of a plate is omitted in calculations and violates it if the dc conductivity is included.

\section{The Casimir-Polder Free Energy at Low Temperature}

We start with the Lifshitz formula for the free energy of Casimir-Polder interaction between both polarizable and magnetizable atom and a ferromagnetic dielectric plate expressed in terms of dimensionless variables ${ }^{14}$

$$
\mathcal{F}(a, T)=\frac{k_{B} T}{8 a^{3}} \sum_{l=0}^{\infty}{ }^{\prime} \Phi\left(\zeta_{l}\right) .
$$

Here, $k_{B}$ is the Boltzman constant, $T$ is temperature of the plate and the environment, $a$ is a distance between the ground state atom and the plate. The prime on the summation sign divides the term $l=0$ by 2 and the function $\Phi$ is defined as

$$
\Phi\left(\zeta_{l}\right)=\Phi^{(\alpha)}\left(\zeta_{l}\right)+\Phi^{(\beta)}\left(\zeta_{l}\right),
$$

where the dimensionless Matsubara frequencies $\zeta_{l}$ are expressed via the dimensional ones as $\zeta_{l}=\xi_{l} / \omega_{c}$, the characteristic frequency is $\omega_{c}=c /(2 a)$ and

$$
\begin{aligned}
& \Phi^{(\alpha)}\left(\zeta_{l}\right)=-\alpha_{l} \int_{\zeta_{l}}^{\infty} \mathrm{d} y \mathrm{e}^{-y}\left[2 y^{2} r_{\mathrm{TM}, l}-\zeta_{l}^{2}\left(r_{\mathrm{TM}, l}+r_{\mathrm{TE}, l}\right)\right], \\
& \Phi^{(\beta)}\left(\zeta_{l}\right)=-\beta_{l} \int_{\zeta_{l}}^{\infty} \mathrm{d} y \mathrm{e}^{-y}\left[2 y^{2} r_{\mathrm{TE}, l}-\zeta_{l}^{2}\left(r_{\mathrm{TM}, l}+r_{\mathrm{TE}, l}\right)\right] .
\end{aligned}
$$

In these equations, $\alpha_{l}=\alpha\left(i \omega_{c} \zeta_{l}\right)$ and $\beta_{l}=\beta\left(i \omega_{c} \zeta_{l}\right)$ are the atomic electric and magnetic polarizabilities. The reflection coefficients for the transverse magnetic (TM) and transverse electric (TE) polarizations of the electromagnetic field are defined as

$$
\begin{array}{r}
r_{\mathrm{TM}, l}=r_{\mathrm{TM}}\left(i \zeta_{l}, y\right)=\frac{\epsilon_{l} y-\sqrt{y^{2}+\zeta_{l}^{2} \gamma_{l}}}{\epsilon_{l} y+\sqrt{y^{2}+\zeta_{l}^{2} \gamma_{l}}}, \\
r_{\mathrm{TE}, l}=r_{\mathrm{TE}}\left(i \zeta_{l}, y\right)=\frac{\mu_{l} y-\sqrt{y^{2}+\zeta_{l}^{2} \gamma_{l}}}{\mu_{l} y+\sqrt{y^{2}+\zeta_{l}^{2} \gamma_{l}}},
\end{array}
$$

where $\gamma_{l}=\epsilon_{l} \mu_{l}-1, \epsilon_{l}=\epsilon\left(i \omega_{c} \zeta_{l}\right)$ and $\mu_{l}=\mu\left(i \omega_{c} \zeta_{l}\right)$ are the dielectric permittivity and magnetic permeability of ferromagnetic dielectric plate at the imaginary Matsubara frequencies. Note that ferromagnetic dielectrics possess physical properties 
characteristic for dielectrics (i.e., low conductivity permitting the use of finite values of $\left.\epsilon_{0}\right)$ but behave like ferromagnets in an external magnetic field! $\frac{15}{[16}$

By means of the Abel-Plana formula, $\frac{14}{14}$ the temperature-dependent contribution to the free energy (11) can be written in the form

$$
\Delta \mathcal{F}(a, T)=\frac{i k_{B} T}{8 a^{3}} \int_{0}^{\infty} \mathrm{d} t \frac{\Phi(i \tau t)-\Phi(-i \tau t)}{\mathrm{e}^{2 \pi t}-1},
$$

where in the above formulas $\zeta_{l}=4 \pi a k_{B} T l /(\hbar c) \equiv \tau l$ should be replaced with $\tau t$.

Now we disregard the dc conductivity of the plate material and consider the frequency-independent permittivity and permeability $\epsilon_{l}=\epsilon_{0}, \mu_{l}=\mu_{0}$. The latter assumption increases by one the power of the primary contribution to the lowtemperature asymptotic expansion of the Casimir-Polder free energy ${ }^{12}[17] \mathrm{We}$ also put $\alpha_{l}=\alpha_{0}$ and $\beta_{l}=\beta_{0}$ which does not influence the primary contribution of this expansion ${ }^{13}$ By putting $\tau t=x$ and introducing the new integration variable $z=y / x$ in Eq. (3), we can rewrite Eq. (2) as

$$
\Phi(x)=-\alpha_{0} I\left(x, \epsilon_{0}\right)-\beta_{0} I\left(x, \mu_{0}\right),
$$

where

$$
I(x, \eta)=x^{3} \int_{1}^{\infty} \mathrm{d} z \mathrm{e}^{-x z}\left[2 z^{2} r(\eta, z)-r\left(\epsilon_{0}, z\right)-r\left(\mu_{0}, z\right)\right]
$$

and

$$
r(\eta, z)=\frac{\eta z-\sqrt{z^{2}+\gamma_{0}}}{\eta z+\sqrt{z^{2}+\gamma_{0}}}
$$

In order to make integrals convergent after putting $x=0$ in the power of an exponent, we subtract two necessary terms from $r(\eta, z)$ and by one from $r\left(\epsilon_{0}, z\right)$ and $r\left(\mu_{0}, z\right)$. To preserve an equality, the same terms are added. As a result, Eq. (7) takes the form

$$
I(x, \eta)=x^{3}\left[I_{1}(x, \eta)+I_{2}(x)+I_{3}(x, \eta)\right],
$$

where

$$
\begin{aligned}
I_{1}(x, \eta) & =2 \int_{1}^{\infty} \mathrm{d} z \mathrm{e}^{-x z}\left\{z^{2}\left[r(\eta, z)-\frac{\eta-1}{\eta+1}\right]+\frac{\eta \gamma_{0}}{(\eta+1)^{2}}\right\}, \\
I_{2}(x) & =\int_{1}^{\infty} \mathrm{d} z \mathrm{e}^{-x z}\left[-r\left(\epsilon_{0}, z\right)+\frac{\epsilon_{0}-1}{\epsilon_{0}+1}-r\left(\mu_{0}, z\right)+\frac{\mu_{0}-1}{\mu_{0}+1}\right], \\
I_{3}(x, \eta) & =\int_{1}^{\infty} \mathrm{d} z \mathrm{e}^{-x z}\left[2 z^{2} \frac{\eta-1}{\eta+1}-A(\eta)\right]
\end{aligned}
$$

and the following notation is introduced:

$$
A(\eta)=\frac{2 \eta \gamma_{0}}{(\eta+1)^{2}}+\frac{\epsilon_{0}-1}{\epsilon_{0}+1}+\frac{\mu_{0}-1}{\mu_{0}+1} .
$$

Calculating $I_{3}$ precisely, for the first terms of expansion in powers of $x$ one obtains

$$
x^{3} I_{3}(x, \eta)=4 \frac{\eta-1}{\eta+1}-A(\eta) x^{2}+\frac{1}{3}\left[3 A(\eta)-2 \frac{\eta-1}{\eta+1}\right] x^{3} .
$$


Calculating $I_{3}$ precisely, for the first terms of expansion in powers of $x$ one obtains

$$
x^{3} I_{3}(x, \eta)=4 \frac{\eta-1}{\eta+1}-A(\eta) x^{2}+\frac{1}{3}\left[3 A(\eta)-2 \frac{\eta-1}{\eta+1}\right] x^{3} .
$$

The first expansion terms of $I_{1}$ and $I_{2}$ are of the order $x^{0}$ and are obtained from Eq. (10) by putting $x=0$ in the powers of exponents using the following integrals:

$$
\begin{aligned}
& \int \mathrm{d} z \frac{\eta z-\sqrt{z^{2}+\gamma_{0}}}{\eta z+\sqrt{z^{2}+\gamma_{0}}}=\frac{1}{\eta^{2}-1}\left[\left(\eta^{2}+1\right) z-2 \eta \sqrt{z^{2}+\gamma_{0}}\right. \\
& \left.-\frac{2 \eta^{2} \sqrt{\gamma_{0}}}{\sqrt{\eta^{2}-1}} \ln \frac{\sqrt{\gamma_{0}}+z \sqrt{\eta^{2}-1}}{\eta \sqrt{\gamma_{0}}+\sqrt{z^{2}+\gamma_{0}} \sqrt{\eta^{2}-1}}\right]+C \\
& \int \mathrm{d} z z^{2} \frac{\eta z-\sqrt{z^{2}+\gamma_{0}}}{\eta z+\sqrt{z^{2}+\gamma_{0}}}=\frac{2}{3\left(\eta^{2}-1\right)^{2}}\left\{3 \gamma_{0} \eta^{2} z+\frac{1}{2}\left(\eta^{4}-1\right) z^{3}-\eta \sqrt{z^{2}+\gamma_{0}}\right. \\
& \left.\times\left[\left(\eta^{2}-1\right) z^{2}+\gamma_{0}\left(\eta^{2}+2\right)\right]-\frac{3 \eta^{2} \gamma_{0}^{3 / 2}}{\sqrt{\eta^{2}-1}} \ln \frac{\sqrt{\gamma_{0}}+z \sqrt{\eta^{2}-1}}{\eta \sqrt{\gamma_{0}}+\sqrt{z^{2}+\gamma_{0}} \sqrt{\eta^{2}-1}}\right\}+C .
\end{aligned}
$$

As a result, for $\Phi(x)$ defined in Eq. (6) we find

$$
\begin{aligned}
\Phi(x)= & -\alpha_{0}\left[4 \frac{\epsilon_{0}-1}{\epsilon_{0}+1}-A\left(\epsilon_{0}\right) x^{2}+B_{\alpha}\left(\epsilon_{0}, \mu_{0}\right) x^{3}\right] \\
& -\beta_{0}\left[4 \frac{\mu_{0}-1}{\mu_{0}+1}-A\left(\mu_{0}\right) x^{2}+B_{\beta}\left(\epsilon_{0}, \mu_{0}\right) x^{3}\right],
\end{aligned}
$$

where

$$
\begin{aligned}
& B_{\alpha}\left(\epsilon_{0}, \mu_{0}\right)=\frac{\mu_{0}^{2}+1}{\mu_{0}^{2}-1}+\frac{\epsilon_{0}^{4}-12 \epsilon_{0}^{2} \gamma_{0}-1}{3\left(\epsilon_{0}^{2}-1\right)^{2}}+\frac{2 \epsilon_{0}^{2} \sqrt{\gamma_{0}}\left(2 \epsilon_{0} \mu_{0}-1-\epsilon_{0}^{2}\right)}{\left(\epsilon_{0}^{2}-1\right)^{5 / 2}} \\
& \times \ln \frac{\sqrt{\gamma_{0}}+\sqrt{\epsilon_{0}^{2}-1}}{\epsilon_{0} \sqrt{\gamma_{0}}+\sqrt{\epsilon_{0} \mu_{0}} \sqrt{\epsilon_{0}^{2}-1}}-2 \sqrt{\epsilon_{0} \mu_{0}}\left[\frac{\epsilon_{0}}{3\left(\epsilon_{0}^{2}-1\right)}+\frac{\mu_{0}}{\mu_{0}^{2}-1}-\frac{2}{3} \frac{\epsilon_{0}\left(\epsilon_{0}^{2}+2\right) \gamma_{0}}{\left(\epsilon^{2}-1\right)^{2}}\right] \\
& -\frac{2 \mu_{0} \sqrt{\gamma_{0}}}{\left(\mu_{0}^{2}-1\right)^{3 / 2}} \ln \frac{\sqrt{\gamma_{0}}+\sqrt{\mu_{0}^{2}-1}}{\mu_{0} \sqrt{\gamma_{0}}+\sqrt{\epsilon_{0} \mu_{0}} \sqrt{\mu_{0}^{2}-1}}
\end{aligned}
$$

and $B_{\beta}\left(\epsilon_{0}, \mu_{0}\right)$ is obtained from $B_{\alpha}\left(\epsilon_{0}, \mu_{0}\right)$ by interchanging $\epsilon_{0}$ and $\mu_{0}$.

Substituting (15) in (5) and integrating, one arrives at

$$
\Delta \mathcal{F}(a, T)=-\frac{\pi^{3}\left(k_{B} T\right)^{4}}{15(\hbar c)^{3}}\left[\alpha_{0} B_{\alpha}\left(\epsilon_{0}, \mu_{0}\right)+\beta_{0} B_{\beta}\left(\epsilon_{0}, \mu_{0}\right)\right] .
$$

It is seen that (17) does not depend on the atom-plate separation. Note that in the limiting case $\beta_{0}=0, \mu \rightarrow 1$ Eq. (16) reduces to

$$
\begin{aligned}
& B_{\alpha}\left(\epsilon_{0}, 1\right)=\frac{\epsilon_{0}-1}{\epsilon_{0}+1} \frac{7 \epsilon_{0}+1}{3\left(\epsilon_{0}+1\right)}-\frac{2 \epsilon_{0}^{2}}{\left(\epsilon_{0}+1\right)^{5 / 2}} \ln \frac{1+\sqrt{\epsilon_{0}+1}}{\sqrt{\epsilon_{0}}\left(\sqrt{\epsilon_{0}}+\sqrt{\epsilon_{0}+1}\right)} \\
& +\frac{\left(\sqrt{\epsilon_{0}}-1\right)\left[\left(3 \epsilon_{0}^{2}+1\right)\left(2 \sqrt{\epsilon_{0}}+1\right)+2 \epsilon_{0}\left(\sqrt{\epsilon_{0}}-1\right)\right]}{3\left(\sqrt{\epsilon_{0}}+1\right)\left(\epsilon_{0}+1\right)^{2}} .
\end{aligned}
$$

This coincides with Eq. (7) in Ref. 13 except that the factor $2 \epsilon_{0}$ in front of the last term in square brackets was indicated in Ref. 13 in error as $3 \epsilon_{0}$. 


\section{The Nernst Heat Theorem and the dc Conductivity}

From Eq. (17) one can calculate the Casimir-Polder entropy at low temperature

$$
S(T)=-\frac{\partial \Delta \mathcal{F}(T)}{\partial T}=\frac{4 \pi^{3}}{15(\hbar c)^{3}} k_{B}\left(k_{B} T\right)^{3}\left[\alpha_{0} B_{\alpha}\left(\epsilon_{0}, \mu_{0}\right)+\beta_{0} B_{\beta}\left(\epsilon_{0}, \mu_{0}\right)\right] .
$$

Thus, $S(0)=0$, i.e., for an ideal ferromagnetic dielectric plate with $\epsilon_{0}<\infty$ and a magnetizable atom the Nernst heat theorem is satisfied. Therefore the Lifshitz theory in this configuration is thermodynamically consistent, provided that the conductivity of ferromagnetic dielectric material at a constant current is disregarded.

Taking into consideration that conductivity of dielectrics, while very small, is really existing effect, one may ask what is its impact on the Casimir-Polder entropy. With account of conductivity the permittivity of plate material $\epsilon_{l}$ is replaced by $\underline{14}$

$$
\widetilde{\epsilon}_{l}=\epsilon_{l}+\frac{4 \pi \widetilde{\sigma}_{0}(T)}{\zeta_{l}}
$$

where the dimensionless conductivity is connected with dimensional by $\tilde{\sigma}_{0}=\sigma_{0} / \omega_{c}$.

It was shown ${ }^{9}$ that the transition from $\epsilon_{l}$ to $\widetilde{\epsilon}_{l}$ leads to only exponentially small in $T$ additions to all terms of the Lifshitz formula with $l \geq 1$. As to the TM reflection coefficient at $l=0$, it takes the values

$$
r_{\mathrm{TM}, 0}=\frac{\epsilon_{0}-1}{\epsilon_{0}+1}, \quad \widetilde{r}_{\mathrm{TM}, 0}=1
$$

when the permittivities $\epsilon_{l}$ and $\widetilde{\epsilon}_{l}$ are substituted in Eq. (4), respectively. In so doing, the reflection coefficient $r_{\mathrm{TE}, 0}$ remains unchanged. Because of this, up to the terms decaying to zero exponentially fast with vanishing temperature, one obtains

$$
\widetilde{\mathcal{F}}(a, T)=\mathcal{F}(a, T)-\frac{k_{B} T}{4 a^{3}}\left(1-\frac{\epsilon_{0}-1}{\epsilon_{0}+1}\right) \alpha_{0},
$$

where $\mathcal{F}$ is found with the dc conductivity disregarded. From this equation we find

$$
\widetilde{S}(a, 0)=\frac{k_{B} \alpha_{0}}{2 a^{3}} \frac{1}{\epsilon_{0}+1}>0 .
$$

Thus, the Casimir-Polder entropy at zero temperature is equal to the positive quantity depending on the parameters of a system (static atomic polarizability, permittivity and atom-plate separation), i.e., the Nernst heat theorem is violated.

\section{Conclusions and Discussion}

We have found the asymptotic behavior at low $T$ of the Casimir-Polder free energy and entropy for a polarizable and magnetizable atom interacting with a ferromagnetic dielectric plate. It is shown that the Nernst theory is satisfied if the dc conductivity of plate material is disregarded and is violated otherwise.

It is of prime importance that the experimental data for both Casimir and Casimir-Polder forces are in agreement with thermodynamically consistent theo-

retical approach disregarding dc conductivity of dielectric materials (see review in 
Ref. 14 and more recent results $\left.\frac{18(19}{19}\right)$. In this respect, it should be mentioned that for graphene systems, where the dielectric response is found basing on the first principles of quantum electrodynamics, the Casimir and Casimir-Polder entropy satisfies the Nernst theorem.20.21 To conclude, thermodynamic problems, arising in application of the Lifshitz theory to real materials, invite further investigation.

\section{Acknowledgments}

V. M. Mostepanenko was partially funded by the Russian Foundation for Basic Research, Grant No. 19-02-00453 A. His work was also partially supported by the Russian Government Program of Competitive Growth of Kazan Federal University.

\section{References}

1. H. Mitter and D. Robaschik, Eur. Phys. J. B 13, 335 (2000).

2. V. B. Bezerra, G. L. Klimchitskaya and V. M. Mostepanenko, Phys. Rev. A 65, 052113 (2002).

3. V. B. Bezerra, G. L. Klimchitskaya and V. M. Mostepanenko, Phys. Rev. A 66, 062112 (2002).

4. V. B. Bezerra, G. L. Klimchitskaya, V. M. Mostepanenko and C. Romero, Phys. Rev. A 69, 022119 (2004).

5. G. L. Klimchitskaya and V. M. Mostepanenko, Phys. Rev. A 95, 012130 (2017).

6. M. Bordag and I. Pirozhenko, Phys. Rev. D 82, 125016 (2010).

7. G. L. Klimchitskaya and C. C. Korikov, Phys. Rev. A 91, 032119 (2015).

8. D. Reiche, K. Busch and F. Intravaia, Quantum Thermodynamics of Overdamped Modes in Local and Spatially Dispersive Materials, arXiv: 1804.01703.

9. B. Geyer, G. L. Klimchitskaya and V. M. Mostepanenko, Phys. Rev. D 72, 085009 (2005).

10. G. L. Klimchitskaya, B. Geyer and V. M. Mostepanenko, J. Phys. A: Math. Gen. 39, 6495 (2006).

11. G. L. Klimchitskaya and V. M. Mostepanenko, J. Phys.: Condens. Matter 29, 275701 (2017).

12. G. L. Klimchitskaya and C. C. Korikov, J. Phys.: Condens. Matter 27, 214007 (2015).

13. G. L. Klimchitskaya, U. Mohideen and V. M. Mostepanenko, J. Phys. A: Math. Theor. 41, 432001 (2008).

14. M. Bordag, G. L. Klimchitskaya, U. Mohideen and V. M. Mostepanenko, Advances in the Casimir Effect (Oxford University Press, 2015).

15. S. A. Chambers, Surf. Sci. Rep. 61, 345 (2006).

16. B. Geyer, G. L. Klimchitskaya and V. M. Mostepanenko, Phys. Rev. B 81, 104101 (2010).

17. G. L. Klimchitskaya and B. Geyer, J. Phys.: Math. Theor. 41, 164032 (2008).

18. C.-C. Chang, A. A. Banishev, G. L. Klimchitskaya, V. M. Mostepanenko and U. Mohideen, Phys. Rev. Lett. 107, 090403 (2011).

19. A. A. Banishev, C.-C. Chang, R. Castillo-Garza, G. L. Klimchitskaya, V. M. Mostepanenko and U. Mohideen, Phys. Rev. B 85, 045436 (2012).

20. V. B. Bezerra, G. L. Klimchitskaya, V. M. Mostepanenko and C. Romero, Phys. Rev. A 94, 042501 (2016).

21. G. L. Klimchitskaya and V. M. Mostepanenko, Phys. Rev. A 98, 032506 (2018). 